\title{
Establishment of a reversible model of prehepatic portal hypertension in rats
}

\author{
XIN ZHAO, JIAN DOU and QING-JUN GAO \\ Department of Hepatobiliary Surgery, The Third Affiliated Hospital of Hebei Medical University, \\ Shijiazhuang, Hebei 050051, P.R. China
}

Received March 26, 2015; Accepted May 3, 2016

DOI: $10.3892 / e t m .2016 .3405$

\begin{abstract}
The aim of the present study was to improve upon the traditional model of pre-hepatic portal hypertension in rats, and simulate the anhepatic phase of orthotopic liver transplantation without veno-venous bypass. A reversible model of portal hypertension was induced by portal vein ligation, with a label ring ligated along the portal vein. A total of 135 male Wistar rats were divided into three groups: i) Normal control (NC) group; ii) portal hypertensive control (PHTC) group; and iii) reperfusion (R) group. In the $\mathrm{R}$ group, rats with portal hypertension underwent simultaneous clamping of the portal triad and retrohepatic vena cava for $1 \mathrm{~h}$, followed by removal of the clamps to enable blood reperfusion. Portal venography and portal vein pressure were recorded during the surgery. Arterial oxygen pressure $\left(\mathrm{PaO}_{2}\right)$, and alanine aminotransferase (ALT), aspartate aminotransferase (AST) and total bilirubin (TBil) levels were determined, and pathological changes of the liver were investigated by immunohistochemical staining. The results demonstrated that, 3 weeks after portal vein ligation, the vein area and the free portal pressures in the PHTC group were significantly increased compared with those in the NC group. The serum ALT and AST levels in the R group at different time points were significantly elevated compared with those in the PHTC group, and reached their maximal levels at $24 \mathrm{~h}$ after reperfusion. Furthermore, the $\mathrm{PaO}_{2}$ at $24 \mathrm{~h}$ after reperfusion was significantly decreased. In conclusion, the reversible model of pre-hepatic portal hypertension in rats was successfully established using the introduction of a label
\end{abstract}

Correspondence to: Professor Jian Dou or Professor Qing-Jun Gao, Department of Hepatobiliary Surgery, The Third Affiliated Hospital of Hebei Medical University, 139 Ziqiang Road, Shijiazhuang, Hebei 050051, P.R. China

E-mail: doujian3rdfy@163.com

E-mail: gaoqingjun_dr@163.com

Abbreviations: $\mathrm{PaO}_{2}$, arterial oxygen pressure; ALT, alanine aminotransferase; AST, aspartate aminotransferase; TBil, total bilirubin

Key words: animal model, portal hypertension, rats, anhepatic phase, liver transplantation ring. This model may be useful for basic research focusing on the anhepatic phase of orthotopic liver transplantation without veno-venous bypass.

\section{Introduction}

Orthotopic liver transplantation has become the primary treatment for patients with end-stage liver diseases (1). It is well known that the high mortality of end-stage liver disease is a global public health problem. The clinical manifestation usually involve cirrhosis caused by different pathological factors, and the prognosis is poor for those with decompensated cirrhosis, which is defined by the presence of ascites, variceal bleeding, encephalopathy and/or jaundice (2). In China, liver failure of the majority of liver transplant recipients is due to hepatitis-induced liver cirrhosis, typically combined with portal hypertension; due to the presence of extensive collateral circulation, in the anhepatic phase, recirculation of blood is possible (3). Therefore, transplantation without veno-venous bypass is the primary type of surgery (3). Currently, problems occur in the anhepatic and post-anhepatic phases, such as hemodynamic instability, metabolic disturbances, and ischemia-reperfusion injuries, which have attracted great attention (4,5). Animal models are important tools with which to investigate these problems, several types of animal models of portal hypertension are currently available, such as drug induced models, and common bile duct ligation models, however, there are a number of major defects in traditional liver transplantation models, such as the inability to reproduce the postoperative recovery phase, high levels of complexity and high mortality rates (6). Therefore, based on the classical partial portal vein ligation method, the aim of the present study was to establish a model by which the whole liver transplantation process, in particular the postoperative recovery phase, could be reproduced.

\section{Materials and methods}

Animals and treatments. 8-week old Male Wistar rats $(\mathrm{n}=135$; 250-300 g body mass) were obtained from the Experimental Animal Center of Hebei Medical University (Shijiazhuang, China) and were housed under the same controlled conditions with a temperature of $25 \pm 2^{\circ} \mathrm{C}$ and a humidity of $50 \pm 10 \%$. Rats were fed with standard laboratory chow and water ad libitum. 
All the experimental procedures were under the approval of the Ethical Committee of the Animal Experiment Center of Hebei Medical University (no. HEBU-2012-02).

Following 1 week of acclimatization, rats were randomly allocated into three groups (15 in each group). Rats in the normal control (NC) group underwent a sham surgery; rats in the portal hypertensive control (PHTC) group underwent partial portal-vein ligation; and rats in the reperfusion $(\mathrm{R})$ group underwent partial portal vein ligation as in the PHTC group, then were subjected to portal vein and inferior vena cava clamping for $1 \mathrm{~h}$, followed by reperfusion for different time periods $(0,6,12,24,48$ and $72 \mathrm{~h}$, and 7 days). Free portal pressure (FPP) was measured and portal venography was performed 3 weeks after portal vein ligation or the sham surgery. Other parameters measured included arterial oxygen pressure $\left(\mathrm{PaO}_{2}\right)$, and biochemical parameters including alanine aminotransferase (ALT), aspartate aminotransferase (AST) and total bilirubin (TBil) expression levels. In addition, pathologic examinations were performed.

Surgical procedure. All groups received a subcutaneous injection of $2 \%$ atropine $(0.05 \mathrm{mg} / \mathrm{kg}$ body mass), and low molecular weight heparin sodium (625 U/kg body mass). After $30 \mathrm{~min}$, rats were anesthetized with $2 \%$ ketamine $(200 \mathrm{mg} / \mathrm{kg}$ body mass; intraperitoneal injection) and a $2 \mathrm{~cm}$ midline abdominal incision was made. In the PHTC and R groups, the portal vein was located and surgically isolated from surrounding tissues. The isolated vessel was stenosed by applying a 3-0 silk thread around the vein, together with an adjacent 20 -gauge blunt-tipped needle and a label ring (made from a 4-F Fogarty embolectomy catheter, with an external diameter of $1 \mathrm{~mm}$ and $\sim 1.5 \mathrm{~cm}$ circumference) lying along the portal vein. After removal of the needle and adjustment of the location of the label ring (in order to prevent its potential vein-twisting effect), the efficacy of partial ligation of the vein was confirmed by observing the increase in the diameter of the vessel prior to ligation, without thrombus or tissue necrosis. Then, the abdominal wall was sutured. Rats in the NC group underwent the same experimental procedure, minus the stenosing of the isolated portal vein. Following surgery, rats were housed for 3 weeks to develop portal hypertension in the corresponding groups.

In the $\mathrm{R}$ group, rats received the same treatment as those in the PHTC group prior to a second surgical procedure conducted 3 weeks after the first surgery. After anesthesia, rats received Ringer's lactate $(5 \mathrm{ml} / \mathrm{h})$ and heparin sodium $(625 \mathrm{U} / \mathrm{kg}$ of body mass) by intubation with a micro-pump in the left external jugular vein; the left carotid artery was intubated and connected with an eight-channel physiological recorder (Beijing Electronic Instrument Factory, Beijing, China) in order to monitor the arterial blood pressure. A $2 \mathrm{~cm}$ incision below the right costal margin was made, and bleeding due to the abdominal wall vein varices was stopped by continuous suture of the surgical margin. The first portal was exposed as indicated by the label ring placed along the portal vein in the first stage surgery, and then the label ring was cut down and removed to restore the portal vein. After exposing the inferior vena cava (above the renal vein, below the liver), the first portal and the inferior vena cava were clamped with non-invasive vascular clamps (Fig. 1A). After $60 \mathrm{~min}$, the clamps were removed and the abdominal wall was sutured.
Portal pressure measurement. Following anesthesia, a 2-cm incision along the right rectus abdominis was made. A segment of the mesenteric branch vein was cannulated with a 3-F epidural catheter (Fig. 1B), and the tip of a $25 \mathrm{~cm}$ catheter was advanced into the trunk of portal vein, and the length inserted was $0.5 \mathrm{~cm}$. The portal pressure was recorded using the pressure transducer of the eight-channel physiological recorder. The spinal value was regarded as zero.

Portal venography. Once the portal pressure had been recorded, a 22-gauge needle was introduced into the splenic pulp and 38\% meglumine diatrizoate was injected through the 3-F epidural catheter using a 22-gauge needle at a rate of $1 \mathrm{ml} / 3 \mathrm{sec}$ (Fig. 1C). Rats in the R group were injected with meglumine diatrizoate again at the same administration rate as before once the label ring had been removed; photographic images were captured and analyzed using the AX-II $500 \mathrm{~mA}$ remote controlled gastrointestinal X-ray machine (Shimadzu Corporation, Kyoto, Japan) and IDR-700 digital X-ray diagnosis system (Shimadzu Corporation).

$\mathrm{PaO}_{2}$ and biochemical parameter analysis. Blood samples $(1 \mathrm{ml})$ from the abdominal aorta were obtained for $\mathrm{PaO}_{2}$ measurement using a blood gas analyzer (Bayer Rapidlab 865; Bayer AG, Fernwald, Germany), and 3-ml blood samples from the inferior vena cava were centrifuged (1,789xg, $5 \mathrm{~min})$. Levels of ALT, AST and TBil in the serum were analyzed using an automatic biochemical analyzer (Olympus AU2700; Olympus Corporation, Tokyo, Japan).

Pathological examination. Anesthesia was performed with $2 \%$ ketamine at a dose of $200 \mathrm{mg} / \mathrm{kg}$. Following anesthesia and sacrifice by cervical dislocation, 3-mm segments of the lower esophagus were dissected from rats 3 weeks after ligation. The left lobe of the liver was dissected from rats 3 weeks after ligation, or at different reperfusion time points $(0,6,12,24,48$ and $72 \mathrm{~h}$, and 7 days). Then, $3-\mu \mathrm{m}$ sections of esophagus or liver tissue were fixed in $10 \%$ buffered formalin, embedded in Paraplast and stained using hematoxylin and eosin (H\&E). Pathological examination was performed using light microscopy (Olympus BX51; Olympus Corporation), images were recorded, and the vein areas in each section of the esophageal mucosa were quantified using the RM6240B/C multichannel bio-signal collection processing system (Chengdu Implement Co., Chengdu, China).

Statistical analysis. Data are expressed as mean \pm standard deviation, and were compared using one-way analysis of variance followed by Bonferroni multiple comparison test. $\mathrm{P}<0.05$ was considered to indicate a statistically significant difference. Statistical calculations were performed using SPSS version 13.0 software (SPSS, Inc., Chicago, IL, USA).

\section{Results}

Gross observations and measurement of the submucosal vein area in the lower esophagus. Three weeks following portal vein ligation, gastric-esophageal vein varices were observed, and the kidneys and spleen were observed to be enlarged and dark red in color. Intestinal wall and mesenteric vein varices 
A

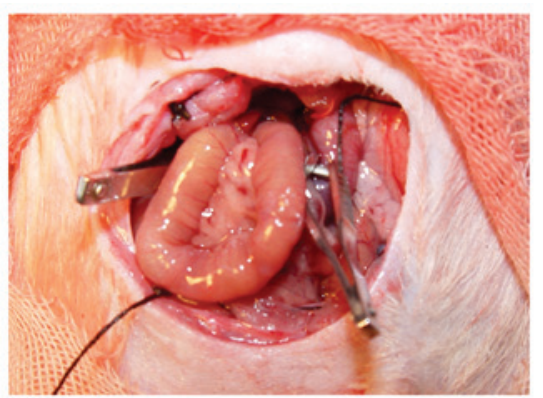

B

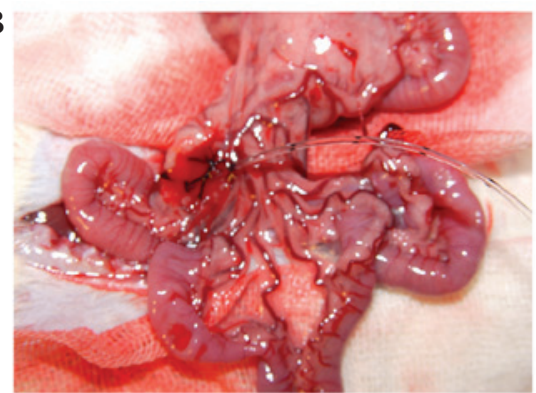

C

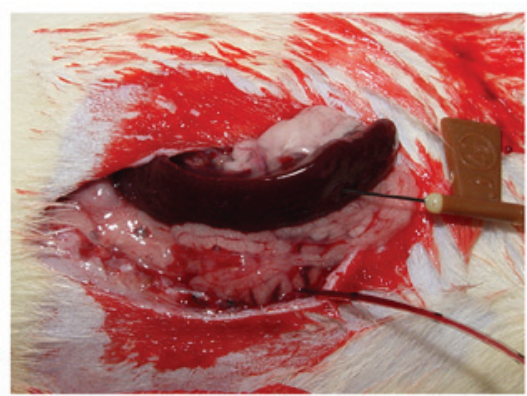

Figure 1. Images of the surgery. (A) Once exposed, the portal vein and inferior vena cava were clamped with non-invasive vascular clamps simultaneously. (B) A segment of the mesenteric branch vein was cannulated with a 3-F epidural catheter in order to measure the portal pressure. (C) Portal venography was performed by splenic pulp puncture.
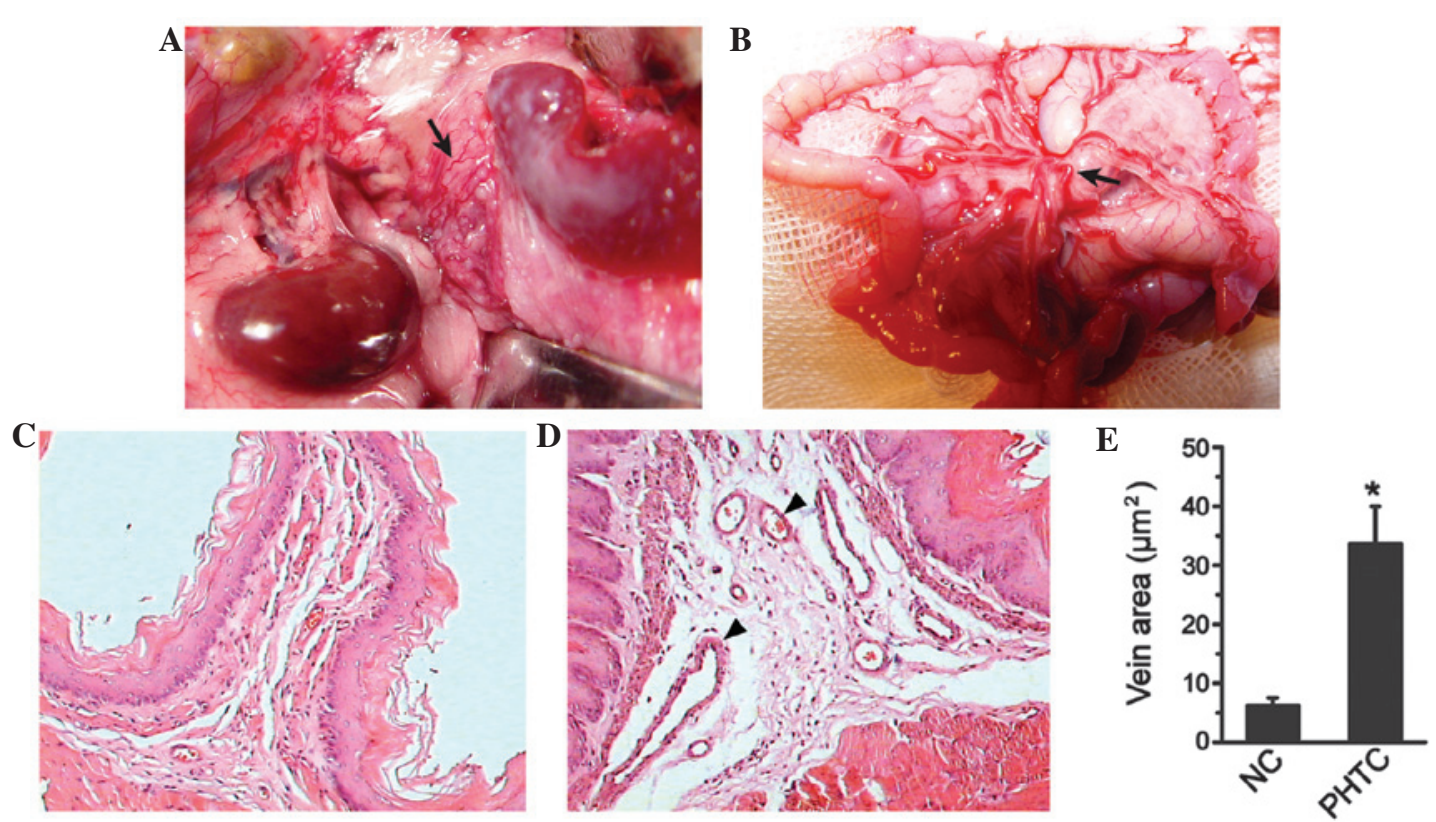

Figure 2. Gross observations and esophagus vein measurement following portal vein ligation. (A) Gastric-esophageal vein varices were observed, and kidneys and spleen were enlarged and in dark red color. (B) Intestinal wall and mesenteric vein varices were also observed, together with edema of the intestinal wall. Hematoxylin and eosin staining was performed on the pathology slides of the esophagus in the (C) NC and (D) PHTC groups. Dilated veins are marked with arrows. (E) The esophagus vein areas were measured in the NC and PHTC groups. "P<0.05 vs. NC. NC, normal control; PHTC, portal hypertensive control.

were also observed, together with edema of the intestinal wall in the PHTC group (Fig. 2A and B). Microscopic examination of the esophagus showed that the submucosal vein in the lower esophagus was dilated in the PHTC group, and the vein area $\left(33.58 \pm 6.42 \mu \mathrm{m}^{2}\right)$ was significantly higher compared with that of the NC group $\left(5.62 \pm 1.30 \mu \mathrm{m}^{2} ; \mathrm{P}<0.05\right.$; Fig. 2C-E).

Portal venography and portal pressure measurement. Portal venography results demonstrated that, in the NC group, the portal vein trunk and its branches were natural in shape, without the presence of portal-systemic collateral circulations (Fig. 3A). In the PHTC group, portal veins were tortuous, and significant narrowing was observed in the vein trunk near the branches (Fig. 3B and C). In the R group, following the removing of the label ring, the constricted vein was restored (Fig. 3D).

The FPP in the NC group was $7.7 \pm 1.7 \mathrm{mmHg}$; while the FPP in the PHTC group with ring was $15.6 \pm 3.1 \mathrm{mmHg}$, which was significantly higher compared with that of the NC group
$(\mathrm{P}<0.05)$. In addition, the FPP in the PHTC group without the ring (measured immediately after the ring was removed) was $13.4 \pm 2.3 \mathrm{mmHg}$, which was significantly higher compared with that of the $\mathrm{NC}$ group $(\mathrm{P}<0.05)$; however, there was no significant difference between the PHTC groups with and without the ring $(\mathrm{P}>0.05$; Fig. 3E).

Serum biochemical parameters and $\mathrm{PaO}_{2}$. There were no significant differences in the serum levels of ALT and AST between the NC group and the PHTC group. However, ALT and AST serum expression levels were significantly increased in the R group after 0 h, 6 h, 12 h, 24 h, $48 \mathrm{~h}$ and $72 \mathrm{~h}$ of reperfusion, compared with the $\mathrm{NC}$ or PHTC groups (all $\mathrm{P}<0.05$ ); the levels of these two enzymes reached their maximum values at $24 \mathrm{~h}$ after reperfusion. At 7 days, the ALT and AST serum levels were decreased and comparable with those of the NC or PHTC groups (Fig. 4A and B). With regards to TBil, there were no significant differences in serum expression levels between all groups (Fig. 4C). In addition, the $\mathrm{PaO}_{2}$ value was 

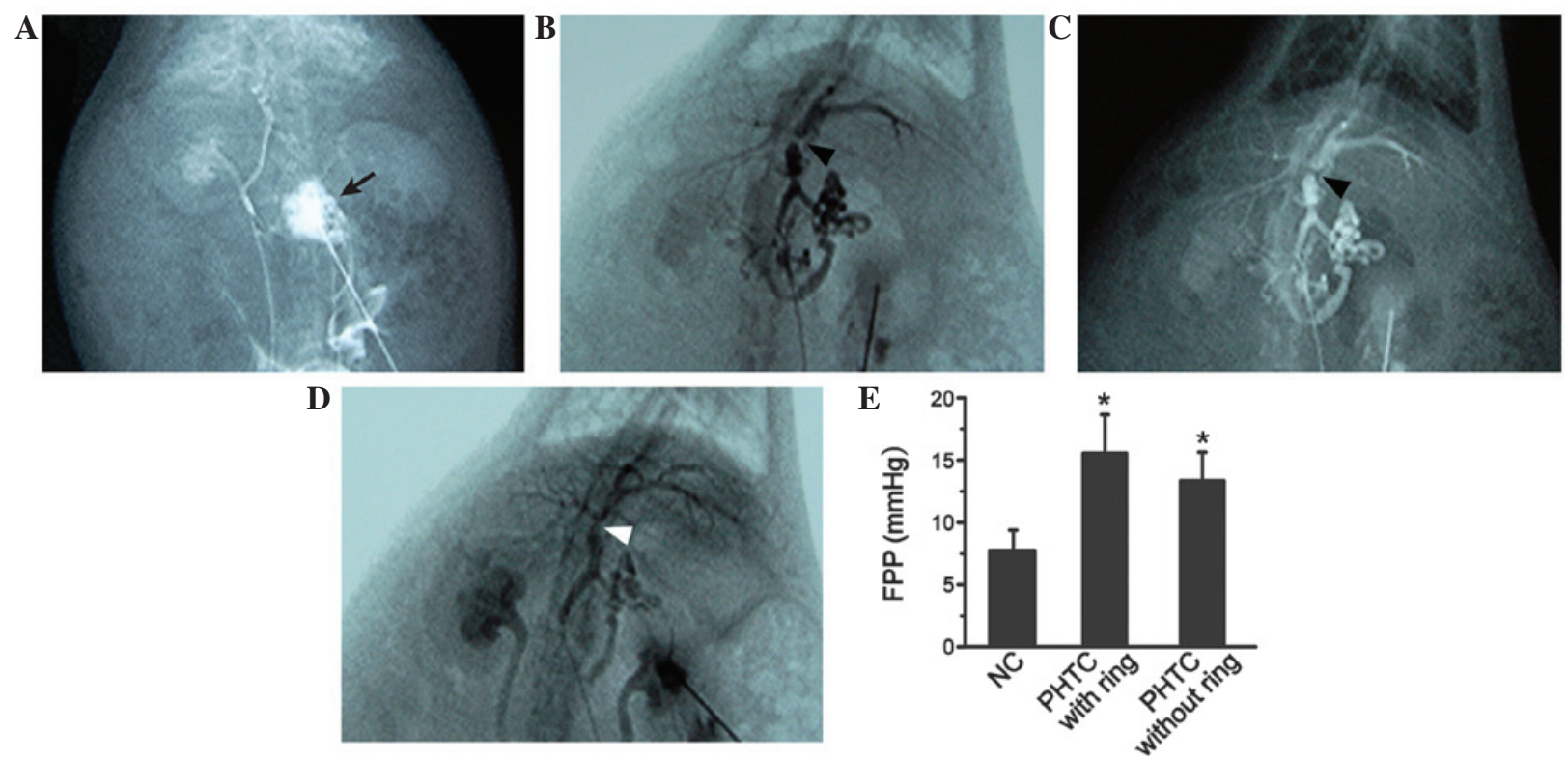

Figure 3. Portal venography and portal pressure measurements. (A) Portal venography in the NC group. Portal venography in the PHTC group (B and C) real time image and developed X-ray film with the ring, respectively, and (D) immediately after the ring was removed. (E) FPP was measured in each group. ${ }^{*} \mathrm{P}<0.05$ vs. NC. The arrows depict (A) the location of splenic vein puncture, (B and C) the implanted label ring and narrowed portal vein and (D) the restored portal vein after the label ring was removed. FPP, free portal pressure; NC, normal control; PHTC, portal hypertensive control.

A

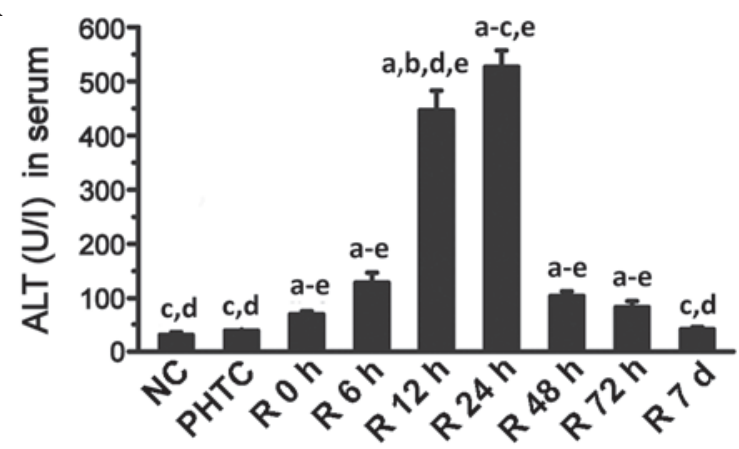

C

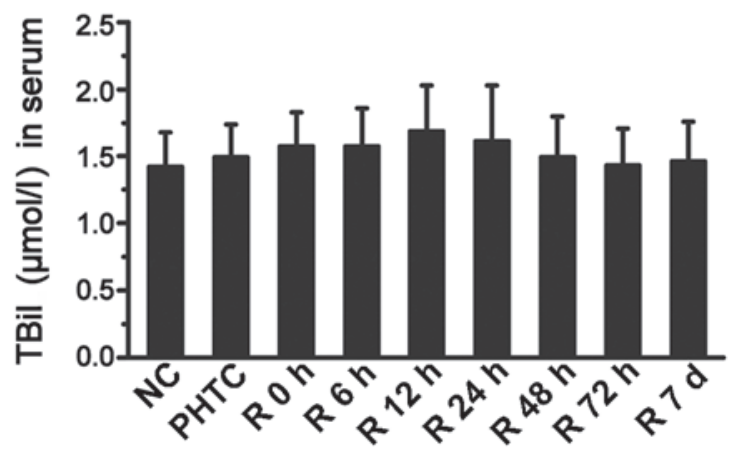

B

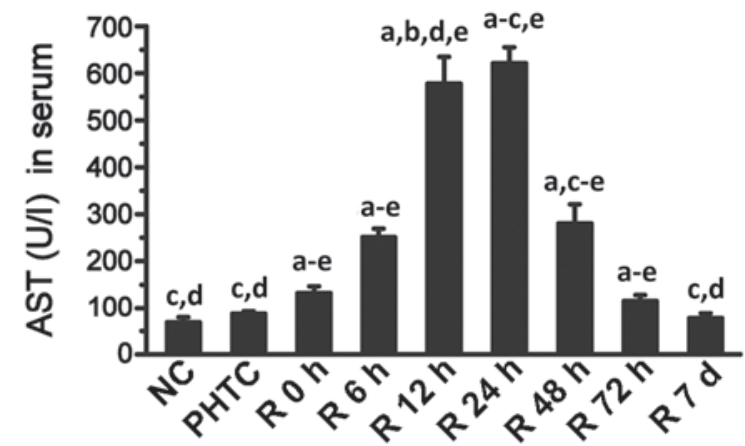

D

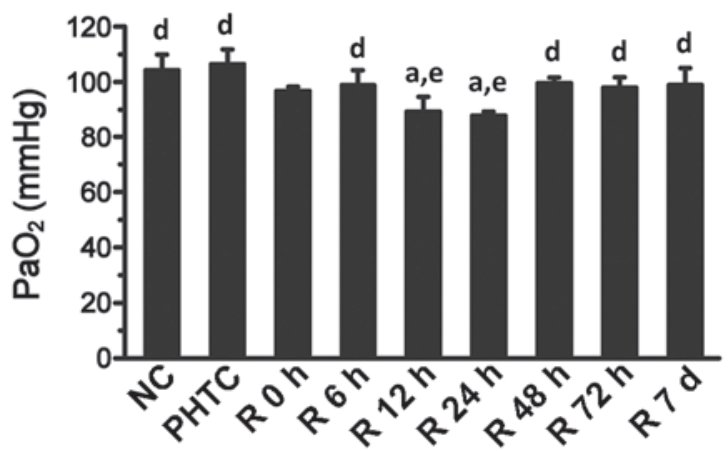

Figure 4. Serum biochemical parameters and $\mathrm{PaO}_{2}$. (A) ALT, (B) AST and (C) TBil serum levels, and (D) $\mathrm{PaO}_{2}$ were measured in the NC, PHTC and R groups. In the $\mathrm{R}$ group, measurements were made at $0,6,12,24,48$ and $72 \mathrm{~h}$, and 7 days (d). ${ }^{\mathrm{a}} \mathrm{P}<0.05 \mathrm{vs}$. NC; ${ }^{\mathrm{P}} \mathrm{P}<0.05 \mathrm{vs}$. PHTC; ${ }^{\mathrm{P}}<0.05 \mathrm{vs}$. $\mathrm{R} 12 \mathrm{~h}$; ${ }^{\mathrm{d}} \mathrm{P}<0.05 \mathrm{vs}$. $\mathrm{R} 24 \mathrm{~h}$; ${ }^{e} \mathrm{P}<0.05$ vs. R 7 d. Values are presented as mean \pm standard deviation $(\mathrm{n}=15)$. ALT, alanine aminotransferase; AST, aspartate aminotransferase; TBil, total bilirubin; $\mathrm{PaO}_{2}$, arterial oxygen pressure; NC, normal control; PHTC, portal hypertensive control; R, reperfusion.

significantly decreased in the R24 h group compared with the NC or PHTC groups (Fig. 4D) (all P<0.05).

Liver pathology examination. H\&E-stained slides were investigated using light microscopy. Results demonstrated that in the NC and PHTC groups, liver cells were well organized, and the hepatic lobules were well-arranged without liver cell edema or neutrophil infiltration. In the $\mathrm{R}$ group at $6 \mathrm{~h}$, the liver cell morphology and structure appeared normal; mild liver cell edema was observed while the hepatic lobule structure was maintained; in the $\mathrm{R}$ group at $12 \mathrm{~h}$, prominent liver cell edema and a small number of infiltrating granulocytes were observed. 
A

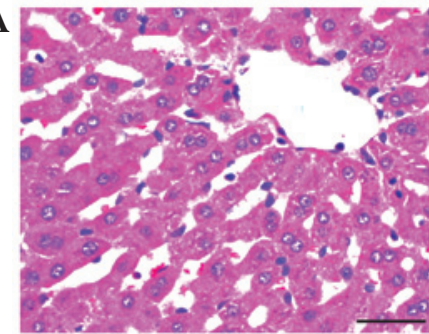

D
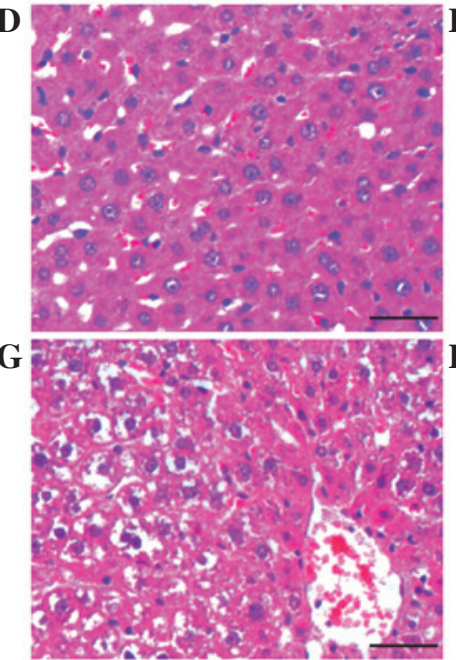
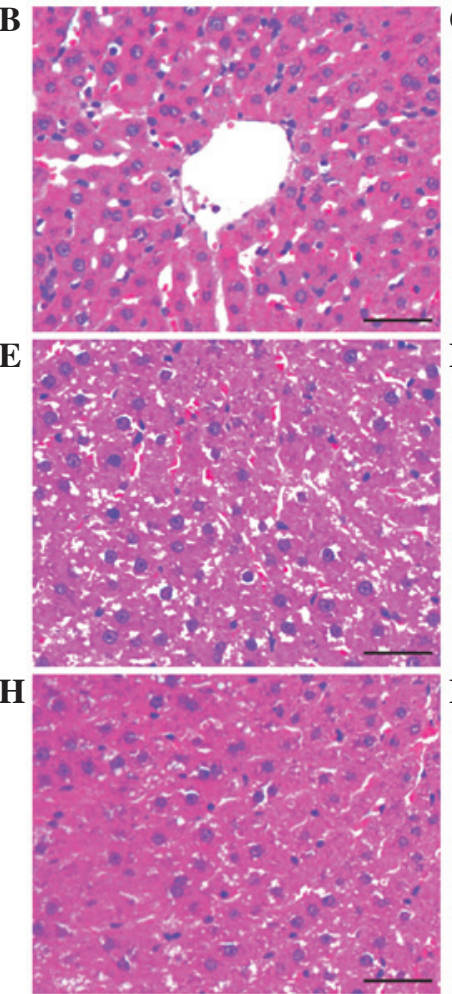
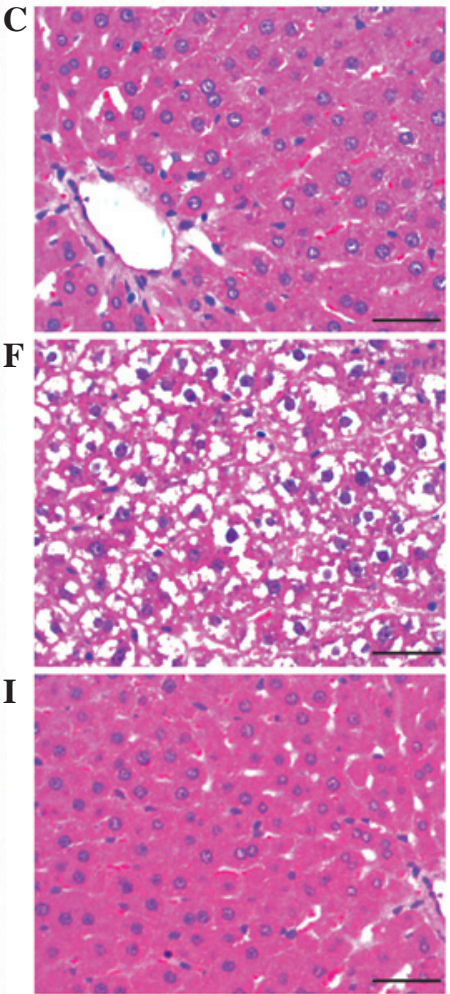

Figure 5. Liver pathology results. Pathological images of liver in the (A) normal control, (B) PHTC and (C-I) R group at (C) 0 h, (D) 6 h, (E) 12 h, (F) 24 h, (G) $48 \mathrm{~h},(\mathrm{H}) 72 \mathrm{~h}$ and (I) 7 days after reperfusion (hematoxylin and eosin staining; scale bar, $50 \mu$ m; magnification, $\mathrm{x} 400$ ).

In the $\mathrm{R}$ group at $24 \mathrm{~h}$, a number of liver cells had become necrotic, and a large quantity of infiltrating granulocytes were observed. These pathological appearances were alleviated in the $\mathrm{R}$ group at $48 \mathrm{~h}$ and $72 \mathrm{~h}$, and the cells were almost restored to normal in the $\mathrm{R}$ group after 7 days (Fig. 5).

\section{Discussion}

Orthotopic liver transplantation without veno-venous bypass, in which the first portal vein and inferior vena cava are clamped simultaneously, has become a mainstream method of surgery in China $(3,5)$. It is advantageous as there is no need to dissect the second portal vein, the liver can be rapidly removed, and complications caused by veno-venous bypass or outflow tract obstruction are avoided. However, the incidence of perioperative complications remains high due to the occurrence of ischemia-reperfusion injury during the process (7). Therefore, it is imperative to establish a model of reversible pre-hepatic portal hypertension and thus to simulate the anhepatic phase of orthotopic liver transplantation without veno-venous bypass, for the purpose of studying different aspects of this process.

Numerous types of animal models of portal hypertension are currently available, including the following: i) Drug induced models, in which carbon tetrachloride (8), thioacetamide (9) or dimethylnitrosamine (10) is used to induce liver injury. Although prominent cirrhosis and portal hypertension are present in these models, portal-systemic collateral circulation is not evident, and longer time periods of induction may be required (11-15 weeks). In addition, portal hypertension is irreversible in these models and thus cannot be used to simulate the liver transplantation process $(11,12)$. ii) Common bile duct ligation models, in which bile duct ligation or dissection, cirrhosis, portal hypertension and portal-systemic collateral circulation can be established in 25-28 days. However, in these models, increases in portal pressure are not evident (13) and no typical pseudolobules are formed in the liver (14). iii) Partial portal vein ligation models, which are easy to reproduce and inexpensive. Presinusoidal portal hypertension develops quickly in these models, and 1 week after portal vein ligation, portal hypertension and portal-systemic collateral circulation can be observed (15). In preliminary experiments for the present study, the use this model was attempted; however, a number of shortcomings were found when this model was used for the purpose of simulating the liver transplantation process. Firstly, heavy adhesion was observed near the first portal vein, which was difficult to dissect, thus the bleeding increased. Secondly, changes to the portal vein were irreversible, despite the ligation being opened. iv) Other models, such as portal hypertension models without liver fibrosis induced by feeding rats with methionine and choline deficient feed (16), and injecting rats with $80-\mu \mathrm{m}$ micro-balls through the portal vein (17). In summary, all of the aforementioned models are not suitable for accurately simulating the process of liver transplantation, including the pre-operative cirrhosis combined with portal hypertension, the intraoperative anhepatic phase and the postoperative portal pressure recovery phase.

Based on the portal vein ligation model, the present study established a reversible model of pre-hepatic portal hypertension in rats. In the first stage of surgery, the trunk of the portal vein was stenosed with an adjacent 20 -gauge blunt-tipped needle and a label ring; then, the needle was removed to partially constrict the portal vein [the diameter of the needle 
and label ring together was $1.9 \mathrm{~mm}$, which is longer than that of the portal vein $(1.5 \mathrm{~mm}-1.8 \mathrm{~mm})]$. In addition to making the portal vein recoverable, the application of a label ring may also be used as a marker, following which the first portal vein can be easily exposed, thus reducing the difficulty of the surgery and reducing the level of bleeding.

The results in the present study demonstrate that, in accordance with classical partial portal vein ligation models, during the first surgical period, the FPP in the PHTC group was significantly elevated compared with that in the NC group, and ascites, varicose epigastric and visceral veins were observed. In addition, the submucosal vein area in the lower esophagus was significantly increased in the PHTC group compared with the NC group. Portal venography results demonstrated that typical portal-systemic collateral circulation had been developed. During the second surgical period, significant ischemia-reperfusion injury in the liver was observed in the histological examination, serum biochemical parameters (ALT, AST and TBil) and arterial $\mathrm{PaO}_{2}$ measurements. In addition, due to the recovery of the constricted portal vein in the $\mathrm{R}$ group, the portal pressure and vein varices were attenuated gradually, and the portal-systemic collateral circulation gradually disappeared. These changes share great similarities with the clinical post-operative state of liver transplantation (18).

There are a number of shortcomings in the present model. Firstly, a certain level of surgical skill is required to perform the number of steps involved in the surgery. Secondly, the introduction of the label ring may increase the incidence of postoperative infection. Finally, typical pseudolobules in the liver may not develop in the model, which limits its application. This is due to the fact that the portal hypertension induced in the model is more evident than drug-induced models or common bile duct ligation models. However, there are still differences between the liver tissues of the portal hypertension model and the realistic portal hypertension, as the latter are usually induced by a chronic pathological stimulus and with typical pseudolobules, whereas, the former is not as usually in an acute condition.

In conclusion, the authors of the current study successfully established a reversible model of pre-hepatic portal hypertension in rats. Thus, the clinical context of orthotopic liver transplantation without veno-venous bypass could be reproduced (as shown in the $\mathrm{R}$ group). This model may be helpful in investigating important mechanisms involved in the process of orthotopic liver transplantation without veno-venous bypass, such as the hemodynamic changes, biochemical parameter alterations, and the non-liver organism injuries involved in the anhepatic and post-anhepatic phases.

\section{Acknowledgements}

This study was supported by a grant from the Key Project of the Health Department of Hebei Province (grant no. 20150286).

\section{References}

1. Kim DY, Huh IY, Cho YW, Park ES, Park SE, Nah YW and Park CR: Experience without using venoveno bypass in adult orthotopic liver transplantation. Korean J Anesthesiol 60: 19-24, 2011.

2. Kim HJ and Lee HW: Important predictor of mortality in patients with end-stage liver disease. Clin Mol Hepatol 19: 105-115, 2013.

3. Chen ZS, Zeng FJ, Ming CS, Lin ZB, Zhang WJ, Wei L, Jiang JP, Zhu XH, Gong NQ, Liu B, et al: Classic orthotopic liver transplantation without venovenous bypass: A report of 45 cases. Transplant Proc 35: 364-365, 2003.

4. Wall WJ, Grant DR, Duff JH, Kutt JL, Ghent CN and Bloch MS: Liver transplantation without venous bypass. Transplantation 43: 56-61, 1987.

5. Wang Y, Liu Y, Han R, Zhu Z, Zhang Y, Wang X, Wang L and Shen Z: Hemostatic variation during perioperative period of orthotopic liver transplantation without venovenous bypass. Thromb Res 122: 161-166, 2008.

6. Abraldes JG, Pasarín M, García-Pagán JC: Animal models of portal hypertension. World J Gastroenterol 12: 6577-6584, 2006.

7. Zhai Y, Petrowsky H, Hong JC, Busuttil RW and Kupiec-Weglinski JW: Ischaemia-reperfusion injury in liver transplantation - from bench to bedside. Nat Rev Gastroenterol Hepatol 10: 79-89, 2013.

8. Deng G, Huang XJ, Luo HW, Huang FZ, Liu XY and Wang YH: Amelioration of carbon tetrachloride-induced cirrhosis and portal hypertension in rat using adenoviral gene transfer of Akt. World J Gastroenterol 19: 7778-7787, 2013.

9. Gao W, Li HY, Wang LX, Hao LJ, Gao JL, Zheng RJ, Cai CJ and Si YL: Protective effect of omeprazole on gastric mucosal of cirrhotic portal hypertension rats. Asian Pac J Trop Med 7: 402-406, 2014

10. Wang XB, Liu P, Tang ZP, Li FH, Liu CH, Hu YY and Xu LM: Cordyceps mycelia extract decreases portal hypertension in rats with dimethylnitrosamine-induced liver cirrhosis: A study on its histological basis. Zhong Xi Yi Jie He Xue Bao 6: 1136-1144, 2008 (In Chinese).

11. Domenicali M, Caraceni P, Giannone F, Baldassarre M, Lucchetti G, Quarta C, Patti C, Catani L, Nanni C, Lemoli RM and Bernardi $\mathrm{M}$ : A novel model of $\mathrm{CCl}_{4}$-induced cirrhosis with ascites in the mouse. J Hepatol 51: 991-999, 2009.

12. Méndez M, Méndez-López M, López L, Aller MA, Arias J and Arias JL: Working memory impairment and reduced hippocampal and prefrontal cortex c-Fos expression in a rat model of cirrhosis. Physiol Behav 95: 302-307, 2008.

13. Van de Casteele M, Sägesser H, Zimmermann H and Reichen J: Characterisation of portal hypertension models by microspheres in anaesthetised rats: A comparison of liver flow. Pharmacol Ther 90: 35-43, 2001.

14. Davies NA, Hodges SJ, Pitsillides AA, Mookerjee RP, Jalan R and Mehdizadeh S: Hepatic guanylate cyclase activity is decreased in a model of cirrhosis: A quantitative cytochemistry study. FEBS Lett 580: 2123-2128, 2006

15. Wen Z, Zhang JZ, Xia HM, Yang CX and Chen YJ: Stability of a rat model of prehepatic portal hypertension caused by partial ligation of the portal vein. World J Gastroenterol 15: 4049-4054, 2009.

16. Francque S, Wamutu S, Chatterjee S, Van Marck E, Herman A, Ramon A, Jung A, Vermeulen W, De Winter B, Pelckmans P and Michielsen P: Non-alcoholic steatohepatitis induces non-fibrosis-related portal hypertension associated with splanchnic vasodilation and signs of a hyperdynamic circulation in vitro and in vivo in a rat model. Liver Int 30: 365-375, 2010.

17. Li XN, Benjamin I and Alexander B: A new rat model of portal hypertension induced by intraportal injection of microspheres. World J Gastroenterol 4: 66-69, 1998.

18. Ito T, Kiuchi T, Yamamoto H, Oike F, Ogura Y, Fujimoto Y, Hirohashi $\mathrm{K}$ and Tanaka AK: Changes in portal venous pressure in the early phase after living donor liver transplantation: pathogenesis and clinical implications. Transplantation 75: 1313-1317, 2003. 